\title{
Bioanalysis
}

\section{An overview of the China Bioanalytical Forum: interview with Daniel Tang}

Daniel Tang talks to Sankeetha Nadarajah, Commissioning Editor (Bioanalysis), regarding the China Bioanalysis Forum (CBF), in which Daniel was one of the co-founders and remains as its co-chair. Daniel is currently the CEO of UP Pharma, a biologics focused bioanalytical CRO in China.

Accepted for publication: 4 January 2016; Published online: 19 January 2017

Keywords: China Bioanalysis Forum • China bioanalysis regulation • GLP

Q Could you tell us a little about yourself \& your role at the China Bioanalytical Forum?

I am the CEO of UP Pharma based in China. I have spent a little over 20 years in the bioanalytical industry, especially in regulatory bioanalysis. Half of my career was spent in the USA in Pharma (Pfizer, among others) after which in 2007, I went back to China. In 2012, a group of scientists decided to set up an organization called the China Bioanalytical Forum, I am one of the co-founders and at the moment remain as co-chair of the organization.

\section{Q What is the focus of the China} Bioanalytical Forum \& its overarching aims?

The focus of the China Bioanalytical Forum (CBF) is to accelerate the advancement of bioanalytical science in China, so we focus on the domestic scientific community. The key aims of the CBF are to:

- Encourage the scientific interaction between the industry and academia in China;

- Promote the harmonization of Chinese bioanalytical regulation with international guidance and also promote execution of the guidance in the community;
- Provide scientific education, technical training and talent development for young scientists.

Q How has the Chinese bioanalytical regulatory landscape evolved in recent times?

I am going to divide the evolution of the Chinese bioanalytical regulatory landscape into three periods in the last 10 years. Before I went back to China in 2007, there were regulations but the implementation of the regulations was not very well done, so in general compliance with regulations was not very good. Between 2007 and 2015, global Pharma and the CROs began to conduct GLP Tox studies in China and as a result, GLP bioanalysis became more popular in China with many global CROs and Pharma beginning to bring the practice to China. During this period, the quality of GLP laboratories ranged, from the worst to the best - the whole spectrum. 22 July 2015 was a key date in the Chinese bioanalytical regulatory landscape because a strict audit from the China Food and Drug Administration on the quality of the clinical trials (including bioanalytical work) was conducted with focus on bioequivalence studies of generic drugs. As a result of this audit, $85 \%$ of studies either was voluntarily withdrawn by the client or sponsor, or was

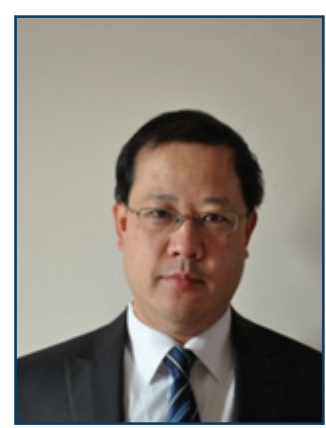

Daniel Tang

United-Power PharmaTech, Beijing, China

danieltang@up-pharma.com

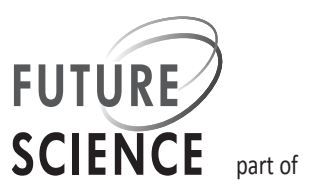


disqualified by the audit. There are many reasons for this result. But as a result, the whole industry began to pay more attention to the quality of our work. So since last July, I would like to think it was a new start for the regulatory landscape of the Chinese bioanalytical world and I can see the quality of bioanalysis will improve substantially over the next few years.

Q Is there a gap between Chinese \& international bioanalytical practice in general: if so, how can this be overcome?

In terms of guidance there is not much difference because the current Chinese Guidance for the Bioanalytical Method Validation is pretty much aligned with the EMA Guidance, however the gap is in the practice. How to interpret and implement the Guidance and putting it into practice is the real issue.

So to overcome this issue, what we are doing at the $\mathrm{CBF}$ is to first organize training courses and conferences for the Chinese scientific community. For example this year the CBF have so far organized three conferences, in which there were training courses. Second, we try to address the issue through use of media (Wechat groups, for example) and technology. We formed groups in Shanghai and Beijing for the young Scientists and we have organized bi-monthly symposiums to discuss different topics from the Guidance and try to form a consensus based on industry practice. Based on this we are looking to publish some White Papers in the future. These are ways the $\mathrm{CBF}$ is trying to overcome this gap.

Q What do you think is most essential for effective \& successful harmonization of Bioanalytical Guidelines globally, across the industry?

I have participated in the initiatives by the Global Bioanalysis Consortium and have had the chance to interact with scientists globally. In my opinion, in order to have the successful harmonization of bioanalytical guidance globally, first we have to promote the exchange of scientific information among the society. For example, collaborations between the CBF, European Bioanalysis Forum (EBF), Japan Bioanalysis Forum and so on, through which information on some of the most advanced technology and knowledge on regulations can be shared. Second, collaboration of different regulatory agencies is very important - the agencies who write the regulations.

Please tell us a little about the progress of the China Pharmacopoeia Guidelines (the first independent BMV guidance in China)

CBF was involved in writing the first BMV guidance for China Pharmacopoeia 2 years ago, which was based on the EMA Guidance. As mentioned earlier these were pretty much aligned; however, we did consider some special issues that are applied to local situations.

For example, when we need to evaluate matrix effects in hemolyzed samples or hypolipidimic sample, we do not make it as a requirement. How to implement and interpret the Guidance is key for us and we are working to overcome the gap with international Guidances. So far, the China Food and Drug Administration has no plan (as far as I know) to publish another guidance and the scientists in China are treating this China Pharmacopoeia Guidelines as the most updated Guidance.

\section{Q Could you tell us about the activities of the} CBF in the past few years?

We have done many things in the last 4 years. First of all, we have our own annual conference, the last one just finished in June 2016 in Nanjing in which we had over 400 participants. Second, our expert committee members participated in the writing of the BMV guidance for the China Pharmacopoeia as mentioned above which is a major milestone for us. Third, we have collaborated with the EBF, AAPS, JBS and so on in the last couple of years. With EBF we organized a workshop called 'Meet the Dragon' 2 years ago in Berlin, Germany, where we discussed the Guidance and import-export issues for clinical sample. We have also worked with AAPS. CBF as a whole provided feedback to the US FDA draft guidance in 2013. Another project we carry out is education among the domestic scientists. We have had training sessions this year and we have bi-monthly seminar for the young scientists in the Beijing area.

\section{Q What can we look forward to from the CBF: what are your upcoming projects?}

In the past 4 years we have established $\mathrm{CBF}$ as the only bioanalytical focus organization in China. We have built up our good reputation among the scientists there. Our focus for the next couple of years will be to:

- Implement international Guidance in China (which will involve a lot of training sessions through conferences and social media). We hope this will also result in us writing some White Papers on different subjects;

- Collaborate with other disciples (e.g., drug metabolism groups in China), and we also look forward to working with other organizations in new areas such as biomarkers and new technology platforms, and with new organizations.

\section{Disclaimer}

The opinions expressed in this interview are those of the interviewee and do not necessarily reflect the views of Future Science Ltd. 


\section{Financial \& competing interests disclosure}

D Tang has no relevant affiliations or financial involvement with any organization or entity with a financial interest in or financial conflict with the subject matter or materials discussed in the manuscript. This includes employment, consultancies, honoraria, stock ownership or options, expert testimony, grants or patents received or pending, or royalties

No writing assistance was utilized in the production of this manuscript. 